\title{
Minimally invasive approach for infective mitral valve endocarditis
}

\author{
Karel M. Van Praet ${ }^{1,2}$, Markus Kofler ${ }^{1}$, Simon H. Sündermann ${ }^{1,2,3}$, Matteo Montagner ${ }^{1}$, Roland Heck ${ }^{1}$, \\ Christoph Starck ${ }^{1}$, Christof Stamm ${ }^{1,2}$, Stephan Jacobs ${ }^{1}$, Jörg Kempfert ${ }^{1}$, Volkmar Falk ${ }^{1,2,3,4}$ \\ ${ }^{1}$ Department of Cardiothoracic and Vascular Surgery, German Heart Center Berlin, Berlin, Germany; ${ }^{2}$ DZHK (German Center for Cardiovascular \\ Research), Partner Site Berlin, Berlin, Germany; ${ }^{3}$ Department of Cardiovascular Surgery, Charité-Universitätsmedizin Berlin, Berlin, Germany; \\ ${ }^{4}$ Berlin Institute of Health (BIH), Berlin, Germany \\ Correspondence to: Karel M. Van Praet, MD. Department of Cardiothoracic and Vascular Surgery, German Heart Center Berlin (Deutsches \\ Herzzentrum Berlin), Augustenburger Platz 1, 13353 Berlin, Germany. Email: vanpraet@dhzb.de.
}

Submitted May 06, 2019. Accepted for publication Jun 18, 2019.

doi: $10.21037 /$ acs.2019.07.01

View this article at: http://dx.doi.org/10.21037/acs.2019.07.01

\section{Clinical vignette}

A 56-year-old Caucasian male patient (STS PROM Score $0.392 \%$; Logistic EuroSCORE I 4.4\%; EuroSCORE II $1.7 \%$ ) was admitted to the neurological ward as an emergency case for a focal neurological deficit. Computed tomography (CT) showed an ischemic event in the area supplied by the middle cerebral artery (MCA). Treatment with lysis therapy and posterior recanalization of the MCA followed and over time neurological symptoms regressed with good results in the control CT. The cause of the embolism was a high-grade mitral valve regurgitation (MR) caused by destroyed leaflets from suspected infective endocarditis (IE). Clinically, our patient experienced a lowgrade fever, arthralgia and myalgia, and a systolic murmur. After serial blood culture sampling, a calculated triple scheme antibiotherapy was started. Enterococcus faecalis could be isolated in the blood culture and, in accordance with the antibiogram, we de-escalated the antibiotherapy to ampicillin and gentamicin. The subsequent blood cultures showed no further growth, febrile episodes had been suspended, and the infection parameters showed signs of regression. A preoperative transesophageal echocardiography (TEE) showed mitral valve (MV) vegetations and a heavy eccentric MR. The left atrium (LA) and left ventricle $(\mathrm{LV})$ were dilated. Due to two positive Duke criteria we diagnosed a bacterial IE of the native MV. Preoperative diagnostic coronary angiography ruled out coronary heart disease. Due to the above-mentioned results we requested urgent minimally invasive $M V$ repair surgery (MIS MVR) and registered our patient for an operation.

\section{Surgical techniques}

\section{Preparation}

\section{Patient positioning and anesthetics}

The patient is intubated with a standard endotracheal tube and positioned supine with a small pillow under the right scapula to elevate the right hemithorax. As endoaortic balloon occlusion cross-clamping is planned, placement of bilateral radial arterial catheters will provide immediate warning of distal displacement of the endoballoon and subsequent innominate arterial obstruction.

\section{Cannulation and cardiopulmonary bypass (CPB)}

The patient is connected to CPB by cannulation of the femoral artery and vein through a $2-\mathrm{cm}$ oblique incision in the groin. TEE is mandatory to confirm the optimum location of the tip of the venous cannula in the superior vena cava (SVC). Body temperature is maintained around $34{ }^{\circ} \mathrm{C}$ and vacuum-assisted venous drainage is used throughout the procedure. In general, a 21 or $23 \mathrm{FR}$ arterial cannula is sufficient depending on patient size and arterial diameter. Introduction of the cannula should be done with TEE confirmation of the endoaortic luminal position of the wire to minimize the risk of dissection or injury. A 25 FR long venous cannula is sufficient and can be positioned at the SVC/right atrium (RA) junction by TEE guidance.

\section{Incisional approach}

A minimally invasive surgery (MIS) periareolar "nipple-cut" approach for surgical MV repair in male patients is used to enter the thorax through the fourth intercostal space. 
It entails a 3 to $4 \mathrm{~cm}$ small convex incision that straddles the right areolar border. A soft-tissue retractor without additional rib-spreading is utilized to expand visibility and entry for the instruments into the thoracic cavity.

\section{Exposition}

\section{Intratboracic exposure}

The right hemidiaphragm is retracted caudally and to the right with a suture placed in tfhe tendinous dome and brought out by a suture hook through a stab incision in the right sixth or seventh space. The pericardium is opened 2-3 cm anteriorly and parallel to the phrenic nerve from the proximal ascending aorta to the diaphragm.

\section{Operation}

\section{Aortic clamping}

Utilizing an aortic endoclamp placed through a side-limb of the femoral arterial CPB-cannula, aortic cross-clamping, antegrade cardioplegia administration, and aortic root venting can be accomplished. The endoclamp is a multilumen catheter with an inflatable balloon at its distal end which provides endo-aortic occlusion. A central lumen can provide antegrade cardioplegia delivery or alternatively aortic root venting. A second tip lumen allows monitoring of aortic root pressure.

\section{Video-assisted fully endoscopic monitoring}

Once the thorax has been entered, a high definition $30^{\circ}$ $3 \mathrm{D}$ thoracoscope is placed into the chest via a $10 \mathrm{~mm}$ port through the second or third intercostal space at the right posterior axillary line. Throughout the procedure, the surgical field is flooded with carbon dioxide $(2 \mathrm{~L} / \mathrm{min})$ through the camera port.

\section{MV exposure and surgical MV repair (1)}

The MV is accessed through a paraseptal incision and a LA retractor is used to expose the MV. In the majority of bacterial endocarditis cases involving the MV a reconstructive valve operation is possible. Should perforation occur, pedicled vegetations can usually be removed followed by pericardial patch closure. Leaflet defects can also be treated by excision of the infected lesion. Limited posterior leaflet involvement is usually handled by triangular resection or quadrangular resection with a sliding plasty. The annular abscess should be treated with a complete sharp excision of the lesions. Whenever the atrio- ventricular or atrio-valvular junctions have been disrupted, reconstruction should be carried out using pericardium or the sliding atrium technique-these complex procedures can be addressed by a minimally invasive approach. Anterior leaflet chordae rupture can be treated by secondary chordae transposition provided that the selected chordae are intact and a few millimeters distant from the resected infected area or by the use of GoreTex neo-chordae. Chordae rupture of the posterior leaflet or the commissures are treated by leaflet triangular or quadrangular resection. Leaflet continuity is then restored either by the sliding leaflet technique or by annular plication. When applying neochordae to the papillary muscles, it is essential that the optimal length and precise location of neochordae in relation to the papillary muscles and the free edge of the mitral leaflets is assessed. The repair is then supported by implanting a semi-rigid annuloplasty ring and antibiotic treatment should be continued for a minimum four to six weeks after surgery (1).

\section{Completion}

\section{De-airing and closure}

After completing the mitral procedure, the vent should be placed across the competent MV to such a depth that venting holes are in both the ventricle and atrium. The LA is then de-aired by starting lung inflation. Epicardial pacing wires should be placed while the heart is still decompressed on CPB. Following this, separation from $\mathrm{CPB}$, decannulation, TEE examination of adequacy of MV repair, and reversal of anticoagulation are all conducted as in a standard operation.

\section{Comments}

\section{Clinical results}

In their textbook Reconstructive Valve Surgery: From Valve Analysis to Valve Reconstruction, Alain Carpentier, David H. Adams and Farzan Filsoufi describe the results of operating at an early stage in the case of native MV IE, i.e., as soon as the organisms had been identified and the antibiotic treatment initiated (1). Indicators for operation included heart failure, severe valvular regurgitation, septic emboli and uncontrolled sepsis. The criteria for performing valve repair were adequate antibiotic therapy for at least one week and large excision of all macroscopically involved tissues (1). In all cases, reconstructive techniques were used. Ring 
annuloplasty was found necessary in $47 \%$ of the patients. Perioperative mortality was $2.5 \%$. Reoperation was necessary in one patient. Late mortality was $2.5 \%$. After a mean followup of 30 months, no recurrence of endocarditis and no reoperation for valvular regurgitation was observed (1-6).

\section{Advantages}

The authors state that they always aim to perform MV reconstruction because it is more resistant to infection than a valvular prosthesis. Early intervention is generally considered when relatively large and mobile vegetations with prior episodes of embolization are present. For patients with IE, MV repair is usually possible and achieves good results. One common advantage of all MIS lateral approaches is the circumvention of a sternotomy and its attendant morbidity. This may be particularly useful in IE, as it would reduce the risk of an additional wound infection.

\section{Caveats}

To ensure the prevention of recurrent endocarditis and/or extension to adjacent structures it is critical that all infected tissue is completely debrided and excised. The use of a MIS technique should not compromise the quality or extent of the operation dictated by the pathological findings (7). Therefore, meticulous preoperative planning and highquality perioperative TEE are paramount. We believe that a prosthetic ring can be used safely if the infection process does not involve the annulus (1). If the infection extends to the annulus, all infected lesions must be excised and excised leaflet tissue should be replaced by pericardium if necessary (1). If we were asked if a head CT scan should be systematically performed in all patients with IE, we would answer that it is mandatory in the presence of clinical neurological manifestations. When considering the optimal timing of surgery following a neurological event, we consider three main factors: (I) the hemodynamic situation and stability of the patient; (II) the type of neurological event (hemorrhagic versus ischemic); (III) mobility and size of the vegetations (1). If the vegetation responsible is large and mobile, therefore increasing the risk of recurrent embolization, then early intervention is recommended (1). If there is further evidence for surgery, such as hemodynamic compromise, surgery should not be postponed (1).

\section{Acknowledgments}

This manuscript was written and produced upon invitation by the Guest Editor and Chief Editor to contribute to the Annals of Cardiothoracic Surgery (ACS) special issue Endocarditis. No financial support and no other potential conflict of interest relevant to this article were reported. The authors would like to thank Susan Holder for editorial assistance.

\section{Footnote}

Conflicts of Interest: The authors have no conflicts of interest to declare.

\section{References}

1. Carpentier AF, Adams DH, Filsoufi F. Carpentier's Reconstructive Valve Surgery: From Valve Analysis to Valve Reconstruction. 1st edition. Elsevier Saunders, 2010.

2. Iung B, Rousseau-Paziaud J, Cormier B, et al. Contemporary results of mitral valve repair for infective endocarditis. J Am Coll Cardiol 2004;43:386-92.

3. Ruttmann E, Legit C, Poelzl G, et al. Mitral valve repair provides improved outcome over replacement in active infective endocarditis. J Thorac Cardiovasc Surg 2005;130:765-71.

4. Zegdi R, Debièche M, Latrémouille C, et al. Longterm results of mitral valve repair in active endocarditis. Circulation 2005;111:2532-6.

5. Pagani FD, Monaghan HL, Deeb GM, et al. Mitral valve reconstruction for active and healed endocarditis. Circulation 1996;94:II133-8.

6. Podesser BK, Rödler S, Hahn R, et al. Mid-term follow up of mitral valve reconstruction due to active infective endocarditis. J Heart Valve Dis 2000;9:335-40.

7. Folkmann S, Seeburger J, Garbade J, et al. Minimally Invasive Mitral Valve Surgery for Mitral Valve Infective Endocarditis. Thorac Cardiovasc Surg 2018;66:525-9.

Cite this article as: Van Praet KM, Kofler M, Sündermann SH, Montagner M, Heck R, Starck C, Stamm C, Jacobs S, Kempfert J, Falk V. Minimally invasive approach for infective mitral valve endocarditis. Ann Cardiothorac Surg 2019;8(6):702704. doi: $10.21037 /$ acs.2019.07.01 